\title{
Crecimiento postnatal y desarrollo del vuelo en el murciélago Leptonycteris yerbabuenae en Chiapas, México
}

\author{
Matías Martínez-Coronel ${ }^{1 *}$, Fernando A. Cervantes ${ }^{2}$ \\ y Yolanda Hortelano-Moncada ${ }^{2}$.
}

Introduction: Neonate bats represent $12-43 \%$ of postpartum-maternal body mass, but unlike other small mammals with large offsprings, bats are altricial and totally dependent on maternal care for survival. Neonates of several bat species are born naked, with low thermoregulatory ability; therefore their growth and development are affected by factors such as humidity and temperature, likewise by sex and food availability, among others. Phyllostomidae is the most diverse family of bats in the Neotropics, nevertheless, there are few studies on the postnatal growth and development of their offspring and especially of glosophagine bats. For that reason, we described the postnatal growth and development of a population of Leptonycteris yerbabuenae inhabiting "The Laguitos" Cave, in the state of Chiapas, México. This species is distributed from the southern United States, through most of Mexico to Guatemala and El Salvador .In Mexico this species is listed as "threatened" by the Mexican government (SEMARNAT, 2010).

Methods: Based on capture-recapture data, we examined the growth trajectories of three morphological variables and flight development of young Leptonycteris yerbabuenae during 1998 and 2001 . Sexual variation, and inter-year variation among specimens of the same age category was assessed by a t Student test. The growth of each variable was measured with a lineal regression analysis, meanwhile the growth parameters were derived from a logistic growth equation and from a polynomial model. To describe the flight development in young bats, we classified these as non-volant, semivolant and volant as was done by Stern et al. (1997) in Phyllostomus hastatus.

Results: The highest records for ambient and roost temperature were in 2001, and so were the lowest records for relative humidity compared with 1998 conditions (Fig. 1). Male and female young bats (Fig. 2) were morphologically similar in the three variables assessed in both years (Table 1). The body mass and length of the forearm increased linearly the first three weeks in both years, while the length of the epiphysis grew until the second week in 1998 and day 10 in 2001, then the growth rate decreased in all variables (Figure 3, Table 2). The sustained flight was achieved at ages 15 and 20 days in 2001 and 1998, respectively (Table 3).

Discussion and conclusions: This study shows that neonates of Leptonycteris yerbabuenae from Chiapas born naked and with the ear meatus closed compared with those of Carbo, Sonora (Gould 1975), what reveals intraspecific variability of these traits. The morphological similarity between male and females young bats was expected; because there is not secondary sexual dimorphism in adults. Neonates of 1998 had lesser body mass and greater length of the fourth metacarpal-phalangeal epiphyseal gap than specimens of 2001. Differences between years were persistent during growth phase, and maybe these results were consequences of different weather conditions that prevailed in each year. The growth parameters derived from the logistic

\footnotetext{
'Departamento de Biología, Universidad Autónoma Metropolitana-Iztapalapa. Avenida San Rafael Atlixco 186, Colonia Vicentina, Iztapalapa, Distrito Federal, México 09340. Email: marti17@ hotmail.com (MM-C)

${ }^{2}$ Departamento de Zoología, Instituto de Biología, Universidad Nacional Autónoma de México. Apartado Postal 70-153, Coyoacán, Distrito Federal, México 04510. Email: fac@ib.unam.mx FAC), yolahm@ib.unam.mx (YH-M)

*Corresponding author
} 
model for forearm length (0.07-0.10) and body mass (0.06-0.10) of $L$. yerbabuenae were similar to other neotropical phyllostomid bats, but lower than vespertilionid bats. These results may be influenced by phylogenetic distance or by environmental conditions. The sustained flight started five days earlier in 2001 compared with 1998, probably influenced by different environmental conditions that prevailed each year. Therefore the sustained flight was achieved at an age similar to those of other phyllostomid bats.

Key words: Cave, Chiroptera, Phyllostomidae, relative humidity, temperature, growth trajectories.

Con base en datos de captura-recaptura se describe el crecimiento postnatal y el desarrollo del vuelo en Leptonycteris yerbabuenae en Chiapas, México. Los murciélagos de esta especie nacen desnudos, con los ojos cerrados y las orejas plegadas. En 1998 tuvieron menor peso y una epífisis cartilaginosa metacarpo-falange del cuarto dedo, más grande en comparación con los de 2001. La longitud del antebrazo y el peso crecieron linealmente los primeros 20 días, con un promedio de 0.85 mm/día en 1998 y de 0.90 mm/día en 2001, después disminuyeron hasta el día 30 a $0.52 \mathrm{~mm} /$ día y a $0.031 \mathrm{~mm} /$ día respectivamente. La longitud de la epífisis tuvo un comportamiento parabólico, en 1998 aumento a una tasa de $0.10 \mathrm{~mm} /$ día los primeros quince días, después disminuyó hasta el día 30 a una tasa de 0.05 mm/día, mientras que en 2001 el incremento fue de $0.10 \mathrm{~mm} /$ día los primeros 10 días y disminuyó hasta el día 30 a una tasa de $0.04 \mathrm{~mm} /$ día. El vuelo sostenido inició a los 15 días en 2001, cuando los juveniles alcanzaron un $84.16 \%$ de la longitud del antebrazo y un $54.59 \%$ del peso del adulto; mientras que en 1998 el vuelo sostenido inició a los 20 dias cuando los juveniles alcanzaron el 83.79\% de la longitud del antebrazo y $49.54 \%$ del peso del adulto. En comparación con otros filostómidos como Artibeus, Carollia y Phyllostomus, los neonatos de L. yerbabuenae nacen con un menor grado de desarrollo y el vuelo sostenido lo consiguen antes de alcanzar la talla del adulto.

Palabras clave: Cueva, Chiroptera, Phyllostomidae, humedad relativa, temperatura, trayectoria de crecimiento.

Introducción

Los murciélagos neonatos son grandes, en comparación con los de otros mamíferos pequeños, pues llegan a representar entre el 12 y el $43 \%$ del peso postparto de la madre (Kurta y Kunz 1987). A diferencia de otros mamíferos que paren crías de gran talla, los neonatos de los murciélagos son altricios y dependen totalmente de la madre para sobrevivir y alimentarse, debido a que son incapaces de volar (Racey y Entwistle 2000) y pueden ser fácilmente depredados. Las crías de varias especies nacen desnudas y con baja capacidad termorreguladora, por lo que son vulnerables a los cambios bruscos de humedad y temperatura (Tuttle y Stevenson 1982). La piel desnuda pierde humedad y calor fácilmente y en caso de bajas temperaturas, el desarrollo y el crecimiento se retrasan.

Por lo tanto, las madres de estas especies de murciélagos deben elegir sitios apropiados para los neonatos, en donde las variaciones ambientales sean mínimas, para disminuir los riesgos de estrés térmico y mortalidad de sus crías. Las cuevas y minas abandonadas 
son refugios idóneos, porque mantienen condiciones ambientales relativamente estables a lo largo del año y son poco accesibles a depredadores potenciales (Kunz 1982).

El crecimiento postnatal de los murciélagos también es afectado por otros factores como el sexo, la disponibilidad de alimento, los cuidados maternales y algunos factores sociales (Tuttle y Stevenson 1982; Hoying y Kunz 1998; Heideman 2000; Kunz y Hood 2000; Porter y Wilkinson 2001). Los estudios sobre crecimiento postnatal en murciélagos se han Ilevado a cabo tanto en cautiverio como en condiciones naturales, aunque la mayoría corresponden a especies que se distribuyen en regiones templadas, principalmente en miembros de la familia Vespertilionidae (Kunz 1987) y recientemente en miembros de las familias Hipposideridae, Molossidae, Phyllostomidae y Thyropteridae, entre otras (Chaverri y Kunz 2006; Krochmal y Sparks 2007; Wyant y Adams 2007; Liu et al. 2009; Lin et al. 2010; Chaverri y Vonhof 2011).

La familia Phyllostomidae es una de las más diversas en los ambientes neotropicales, sin embargo existen pocos trabajos sobre el desarrollo posnatal de sus crías. Sólo se han publicado reportes sobre el crecimiento postnatal de algunas especies como los murciélagos fruteros Artibeus jamaicensis, A. watsoni y Carollia perspicillata, el vampiro común Desmodus rotundus y los murciélagos carnívoros Phyllostomus discolor y $P$. hastatus (Kleiman y Davies 1979; Kunz y Hood 2000; Chaverri y Kunz 2006). En estos estudios se ha observado, por ejemplo, que en $A$. jamaicensis las crías nacen con los ojos abiertos y el cuerpo cubierto de pelo. Las orejas se tornan erectas durante las primeras seis horas de vida y que representan el $31 \%$ del peso y el $55 \%$ de la longitud del antebrazo del adulto (Taft y Handley 1991). De manera similar, en A. watsoni los neonatos representan el 32 y $52 \%$ del peso y de la longitud el antebrazo de la madre, respectivamente, y que son capaces de volar después de los 35 días, cuando han alcanzado el $100 \%$ de la longitud del antebrazo y el $80 \%$ del peso del adulto. Sin embargo, se desconoce casi por completo los detalles de la biología reproductiva de murciélagos consumidores de néctar y polen. Por esta razón, en el presente trabajo se reporta el desarrollo y el crecimiento postnatal de una población de Leptonycteris yerbabuenae que habita en la cueva de Los Laguitos, Chiapas.

Leptonycteris yerbabuenae es un murciélago con una distribución geográfica amplia que incluye el sur de los Estados Unidos de América, casi todo México hasta Guatemala y El Salvador (Arita y Humphrey 1988). Este murciélago habita en los matorrales xerófilos, cardonales y selva baja caducifolia, donde obtiene su alimento principal, que consiste en néctar y polen de cactáceas, agaváceas, fabáceas y bombacáceas (Alcorn et al. 1961; Alcorn 1962; Álvarez y González-Quintero 1969; Quiroz et al. 1986; Cockrum y Petryszyn 1991; Fleming et al. 1993; Riechers-Pérez et al. 2003) entre otras. Esta especie es considerada como cavernícola obligada (Arita 1993). En México la especie está catalogada en la categoría de "amenazada" de extinción por el gobierno mexicano, bajo la sinonimia de L. curasoae (SEMARNAT- 2010), se considera que la constante perturbación de su hábitat es una de las principales causas que contribuyen a esta condición.

Área de estudio. El estudio del desarrollo y crecimiento postnatal de L. yerbabuenae se Ilevó a cabo en la cueva de Los Laguitos, que se localiza a 4 km NW de la ciudad de 
Tuxtla Gutiérrez, municipio del mismo nombre, Chiapas, a $781 \mathrm{~m}$ sobre el nivel del mar $\left(16.778333^{\circ} \mathrm{N}, 93.148611^{\circ} \mathrm{W}\right)$. La temperatura promedio de la cueva es de $32.3^{\circ} \mathrm{C}$ y la humedad relativa promedio es de $95.4 \%$, por lo que se clasifica como una "cueva de calor" (De la Cruz 1992; Martínez-Coronel et al. 2010). El clima de la zona corresponde a un clima cálido subhúmedo con lluvias en verano, la temperatura media anual es de $24.7^{\circ} \mathrm{C}$ (Awo"(w)(i')g; Cardoso 1979). La vegetación que rodea a la cueva corresponde a un bosque tropical caducifolio (Miranda 1998) con cambios fenológicos drásticos entre la estación húmeda y la seca. Durante el tiempo en que se desarrolló el trabajo la cueva estuvo habitada por otras especies de murciélagos como son: Balantiopteryx plicata, Mormoops megalophylla, Pteronotus davyi, P. parnelli, P. personatus, Artibeus jamaicensis, Glossophaga soricina y Natalus stramineus.

La cueva tiene un desarrollo horizontal, que consta de una sola entrada y un túnel principal que corre en dirección noreste, al cual se unen otros dos túneles a $65 \mathrm{~m}$ de la entrada, uno del lado norte y otro del lado sur, que en conjunto suman una longitud mayor a $600 \mathrm{~m}$. La temperatura y la HR de la cueva varían entre las diferentes secciones de cada túnel a lo largo del día y entre meses a lo largo del año, dependiendo de la topografía del terreno y de la abundancia de las poblaciones de murciélagos (MartínezCoronel et al. 2010).

Para determinar el momento en el que iniciaban los nacimientos, se visitó diariamente la cueva de Los Laguitos desde el 15 de octubre de 1998 y de 2001. En 1998 los primeros nacimientos se observaron el 24 de octubre, por lo que el monitoreo se llevó a cabo del 26 de octubre al 26 de noviembre, mientras que en el 2001 los primeros nacimientos sucedieron el 22 de octubre y el monitoreo se llevó a cabo del 24 de octubre al 28 de noviembre.

Los sitios para el estudio fueron seleccionados con base en la accesibilidad a los neonatos. Estos sitios se ubicaron en la primera sección del túnel situado al norte del túnel principal, que corresponde a una galería que tiene $85 \mathrm{~m}$ de longitud, $25 \mathrm{~m}$ de ancho y 30 m de alto, cuyo piso está cubierto por rocas de más de $1 \mathrm{~m}$ de diámetro y paredes erosionadas. Los sitios elegidos se ubicaron en paredes con una altura máxima de $3 \mathrm{~m}$, las que en visitas previas se detectó que son usadas por las hembras de $L$. yerbabuenae para dejar a sus críos durante su salida nocturna. Algunas hembras usan sitios de percha para sus críos muy cerca del piso, la distancia mínima que se detecto fue de $30 \mathrm{~cm}$ de altura.

Se midió la temperatura y la humedad relativa (HR) con ayuda de un Psicrómetro Taylor de mercurio de bulbo húmedo y bulbo seco (con $\pm 5 \%$ de precisión y $2 \%$ de resolución para la HR y con $\pm 3{ }^{\circ} \mathrm{C}$ de precisión y $1{ }^{\circ} \mathrm{C}$ de resolución para la temperatura). Estas variables se registraron en cuatro sitios diferentes, que correspondieron con la ubicación de los neonatos estudiados. En cada uno de ellos se tomaron dos lecturas de la temperatura y $\mathrm{HR}$, una a las 19:00 cuando las madres dejaban a su crío y otra a las 23:00 h cuando regresaban, ambas a $1.20 \mathrm{~m}$ de altura a un lado de las paredes donde perchan los neonatos. Para evaluar las diferencias de temperatura y HR entre años, se aplicó una prueba de $t$ de Student (Zar 1996).

Para determinar el crecimiento de los neonatos de $L$. yerbabuenae, cada individuo fue marcado con anillos de plástico numerados, de $5 \mathrm{~mm}$ de diámetro y $5 \mathrm{~mm}$ de ancho. Se colocó la marca en el antebrazo izquierdo en las hembras y en el derecho a los machos. 
Todos los ejemplares anillados tenían el cordón umbilical, la placenta fresca, el cuerpo arrugado y la piel de color rosado, criterios que se han usado como características de individuos recién nacidos o de un día de edad (Kunz y Anthony 1982; Stern y Kunz 1998).

El horario de trabajo fue de las 19:00 a las 23:00 h, aprovechando que durante este tiempo las madres salían para alimentarse y dejaban a su crío en el sitio de percha. No fue posible continuar las observaciones después de ese horario, debido a que ellas regresaban para amamantarlos y los cambiaban de lugar. Los ejemplares fueron manipulados a un lado de su sitio de percha y en el menor tiempo posible (normalmente menos de 5 minutos). Al finalizar la medición y la observación de cada neonato y antes de ubicarlo en su sitio de percha original, se midió el desarrollo de su capacidad de vuelo, para lo cual el individuo se sostuvo de sus patas traseras con los dedos índice y medio de la mano del investigador y mediante movimientos verticales bruscos se le incito a volar. Para describir el desarrollo del vuelo se emplearon las categorías que Stern et al. (1997) definieron para Phyllotomus hastatus, y que son las siguientes: a) no volantones, individuos que colgados del dedo o de las paredes rehúsan volar, permanecen quietos en su sitio de percha o sólo se mueven por las paredes; b) semivolantones, individuos que intentan el vuelo pero no consiguen mantenerse, chocan contra las paredes de la cueva o no logran perchar y c) volantones, individuos capaces de volar en línea recta y que logran perchar. Durante la manipulación ningún individuo murió, posiblemente por tratarse de una especie menos inquieta en comparación con otros filostómidos como Artibeus jamaicensis.

El crecimiento se valoró a través de los cambios que experimentaron en el peso, la longitud del antebrazo derecho y la longitud de la epífisis cartilaginosa de la unión metacarpo-falange del $4^{\circ}$ dedo del ala derecha, variables que fueron medidas cada cinco días en los individuos recuperados (Kunz y Anthony 1982). Las variables longitudinales se registraron en milímetros y fueron tomadas con un vernier digital (Mitutoyo con precisión de $0.01 \mathrm{~mm}$ ), mientras que el peso fue expresado en gramos y fue tomado con una balanza digital (Ohaus, con precisión de $0.1 \mathrm{~g}$ ). De cada variable fueron calculados la media y el error estándar para cada categoría de edad. Las categorías de edad empleadas fueron las siguientes: 1, 5, 10, 15, 20, 25, 30 y 35 que corresponden a la edad cronológica de los individuos expresada en días.

Las diferencias debidas al sexo y las debidas al año de muestreo para individuos de la misma edad se evaluaron con una prueba de $t$ de Student. Aun cuando el tamaño de muestra es pequeño para las variables analizadas en algunas categorías de edad, estas cumplieron con los supuestos estadísticos que exige un análisis paramétrico (Zar 1996), excepto para la categoría de edad 30 días de ambos años en el análisis de dimorfismo sexual. No obstante, se decidió utilizar ésta prueba, debido a que los resultados obtenidos fueron similares a los encontrados en las demás categorías de edad. Los cambios debidos a la edad, fueron estimados para cada una de las variables por medio de una regresión lineal, en la parte lineal de cada curva. Para determinar las diferencias entre años, se comparó la pendiente y elevación de cada curva (en su parte lineal) con una prueba de $t$ de Student modificada (Zar 1996).

Los datos de la longitud del antebrazo y el peso contra la edad, se emplearon para derivar los parámetros de crecimiento de $L$. yerbabuenae, a través del modelo logístico 
convencional (Zullinger et al. 1984; Kunz y Hood 2000), de acuerdo con la siguiente ecuación:

$$
M(t)=A\left\{e^{-K(t-1)}+1\right\}^{-1}
$$

dónde: $\mathrm{M}(\mathrm{t})$ = valor de la variable (antebrazo o peso) a la edad t (días), A = valor asintótico de la variable (antebrazo o peso), $\mathrm{K}=$ constante de la tasa de crecimiento (días ${ }^{-1}$ ) e I = edad del punto de inflexión (días). Los modelos de Gomperz y von Bertalanffy comúnmente usados en otros estudios también fueron aplicados (Zullinger et al. 1984), pero su ajuste fue menor al del modelo logístico, razón por la cual no se presentan estos resultados. Por otra parte, la relación entre la longitud cartilaginosa de la epífisis del cuarto dedo y la edad, se ajustó a un modelo polinomial. Los análisis fueron realizados con el programa Number Crunching Statistical System que emplea el algoritmo de Marquardt-Levenverg (Hintze 2007).

Ejemplares examinados. Se marcaron un total de 118 neonatos, 57 en 1998 y 61 en el 2001. La información sobre el crecimiento está basada en los ejemplares que fueron recapturados al menos tres veces. En 1998 fueron 17 machos y 20 hembras, mientras que en el 2001 fueron 20 machos y 17 hembras.

Condiciones del ambiente cavernícola. Las condiciones ambientales del interior del refugio fueron diferentes en los dos períodos de estudio; la temperatura promedio ambiental fue menor en $1998\left(\bar{X}=33.29{ }^{\circ} \mathrm{C} \pm 0.18\right.$, intervalo $32.0-35.0{ }^{\circ} \mathrm{C}$ ) en comparación con el $2001\left(\overline{\mathrm{X}}=34.04{ }^{\circ} \mathrm{C} \pm 0.08,33.0-35.0{ }^{\circ} \mathrm{C} ; \mathrm{t}=8.55, P=0.0000\right)$; en cambio la humedad relativa fue mayor en el primer año ( $\mathrm{HR}=100 \%)$ con relación al segundo $(\mathrm{HR}=94.81 \pm 0.35,93.0-100 \% ; \mathrm{t}=14.64, P=0.0000$ (Fig. 1). Estas condiciones se relacionan positivamente con las del ambiente externo, ya que 1998 fue un año con menor temperatura media anual $\left(22.8^{\circ} \mathrm{C}\right)$ pero con mayor precipitación $(1,074.7 \mathrm{~mm})$, mientras que en el 2001 la temperatura fue mayor $\left(25.7^{\circ} \mathrm{C}\right)$ y con menor precipitación (827.6 mm; Estación meteorológica "Tuxtla Gutiérrez", $16.752777^{\circ}$ N; $93.116666^{\circ} \mathrm{W}$, clave 00007202 CONAGUA).

Características de los neonatos. Los recién nacidos se caracterizaron por tener la placenta unida, la piel arrugada, de tonalidad rosada y desnuda, excepto en la barbilla y entre las piernas donde presentaban escaso pelo de una longitud menor a $1 \mathrm{~mm}$. Las orejas estaban plegadas a los lados de la cabeza y el meato auditivo cerrado. Los ojos estaban cubiertos por los párpados, de los que solamente se observaba la línea de unión (Fig. 2). El neonato no se desplazaba del sitio donde lo dejaba la madre, tan solo emitía chillidos audibles y movía la cabeza constantemente ante cualquier sonido, ya sea del aleteo de otros murciélagos o el provocado intencionalmente por nosotros.

De las tres variables morfométricas analizadas, ninguna mostró diferencias significativas debidas al sexo en 1998 (longitud del antebrazo, hembras $(\mathrm{h}): \overline{\mathrm{X}} \mathrm{h}=26.74$ \pm 0.55 , machos $(\mathrm{m}): \overline{\mathrm{X}} \mathrm{m}=25.59 \pm 0.44 ; \mathrm{t}_{35 \text { g.l. }}=1.50, \mathrm{P}=0.12 ;$ peso, $\overline{\mathrm{X}} \mathrm{h}=5.25 \pm 0.16$, $\overline{\mathrm{X}} \mathrm{m}=5.51 \pm 0.12 ; \mathrm{t}_{35 \text { g.l. }}=1.23, \mathrm{P}=0.22$; longitud de la epífisis, $\overline{\mathrm{X}} \mathrm{h}=4.60 \pm 0.08, \overline{\mathrm{X}} \mathrm{m}$ $=4.59 \pm 0.07, \mathrm{t}_{35 \text { g.l. }}=0.02, \mathrm{P}=0.97$ ) ni en 2001 (longitud del antebrazo, $\overline{\mathrm{X}} \mathrm{h}=27.40 \pm$ 
0.33, $\overline{\mathrm{X}}_{\mathrm{m}}=26.69 \pm 0.31 ; \mathrm{t}_{35 \text { g.l. }}=1.52, \mathrm{P}=0.13 ;$ peso, $\overline{\mathrm{X}} \mathrm{h}=6.28 \pm 0.16, \overline{\mathrm{X}} \mathrm{m}=6.55 \pm$ $0.18 ; t_{35 \text { g.l. }}=1.05, P=0.30$; longitud de la epífisis, $\bar{X} h=3.69 \pm 0.06, \bar{X} m=3.88 \pm 0.07$; $\mathrm{t}_{35 \mathrm{~g} . .^{\circ}}=1.83, \mathrm{P}=0.07$ ). Con base en estos resultados, los sexos fueron agrupados para su comparación entre años.

La comparación entre años mostró que los neonatos de 1998 fueron significativamente menos pesados que los de $2001\left(\overline{\mathrm{X}}_{1998}=5.37 \pm 0.10 ; \overline{\mathrm{X}}_{2001}=6.42 \pm 0.12 ; \mathrm{t}_{72 \text { g.l. }}=6.50, \mathrm{P}\right.$ $=0.0000)$ pero con una longitud de la epífisis cartilaginosa significativamente mayor en $1998\left(\bar{X}_{1998}=4.59 \pm 0.05 ; \bar{X}_{2001}=3.79 \pm 0.05 ; t_{72 \text { g.l. }}=10.82, P=0.0000\right)$, en cambio en la longitud del antebrazo las diferencias no fueron significativas entre ambos años $\left(\bar{X}_{1998}\right.$ $=26.21 \pm 2.27 ; \overline{\mathrm{X}}_{2001}=27.02 \pm 1.43 ; \mathrm{t}_{72 \mathrm{~g} . \mathrm{l}}=1.83, \mathrm{P}=0.07$ ).

En comparación con los adultos, los neonatos del 2001 tuvieron proporcionalmente una longitud del antebrazo mayor (49.26\%) y fueron más pesados (26.90\%) que los de 1998 (47.60\% y $20.58 \%$ respectivamente).

Figura 1. Trayectorias de la temperatura (a) y la humedad relativa (b) que prevalecieron durante el crecimiento de los juveniles de Leptonycteris yerbabuenae en la cueva de Los Laguitos, Chiapas. El punto se refiere al valor medio, el recuadro al error estándar y las líneas a los valores extremos.
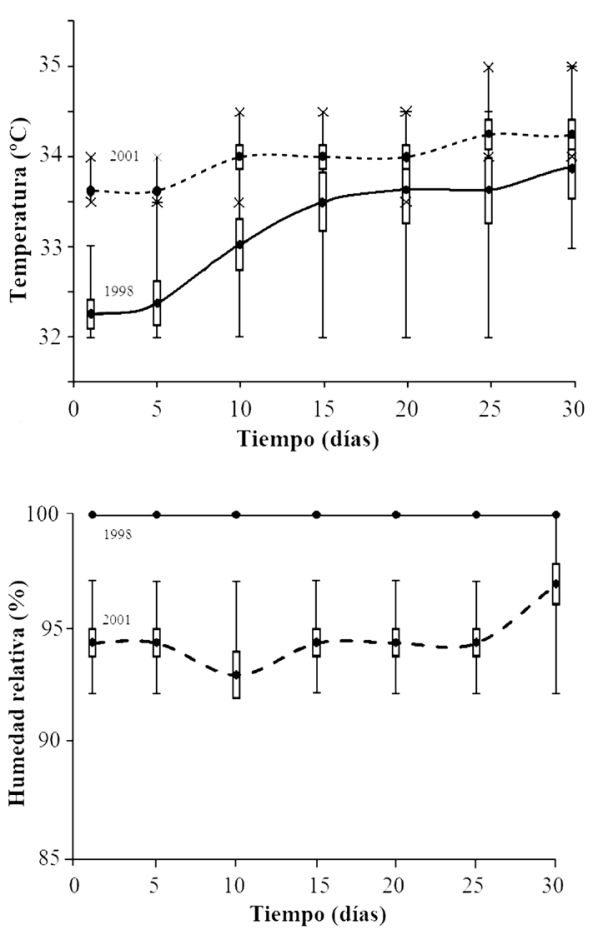

Dimorfismo sexual y crecimiento.

La ausencia de diferencias significativas debidas al sexo encontrada en los neonatos se repitió en las seis categorías de edad restantes (5, 10, 15, 20, 25 y 30 días) en las tres variables analizadas (longitud del antebrazo, peso y longitud de la epífisis del cuarto dedo), con excepción de las categorías de edad 15 y 20 de la longitud de la epífisis de la muestra de 2001 (Tabla 1). Debido a que éste comportamiento no se repitió en las demás categorías de edad de 2001 ni en las de 1998, las muestras de ambos sexos se agruparon por categoría de edad para llevar a cabo el siguiente análisis.

La comparación entre años reveló la presencia de diferencias significativas entre las muestras de 1998 y 2001. Se encontró que en la longitud del antebrazo, los individuos del 2001 fueron significativamente mayores a los de 1998 durante los primeros 20 días, 
después las diferencias dejaron de ser significativas para las categorías de edad 25 y 30 (Tabla 2). En cuanto al crecimiento, se observó que la longitud del antebrazo aumentó de manera constante y fue casi lineal los primeros 20 días en ambos años de estudio, con una tasa de incremento de 0.85 mm/día en 1998 y de 0.90 mm/día en 2001 (Fig. 3 a y b). De los días 20 a 30, la tasa de crecimiento disminuyó a 0.52 mm/día en 1998; y a $0.31 \mathrm{~mm} /$ día en el 2001. Al comparar la curva de crecimiento de 1998 contra la de 2001, resultó que no fueron diferentes en los primeros 20 días (pendiente: $\mathrm{t}_{(2) 317 \text { g.l. }}=$ $0.36, P>0.05$; elevación $\mathrm{t}_{(2) 317 \text { g.l. }}=0.98 .53, P>0.05$ ) ni del día 20 al 30 (pendiente: $\mathrm{t}_{(2) 93}$ $=1.21, P>0.05$, elevación: $\left.\mathrm{t}_{(2) 93 \text { g.l. }}=1.86, P>0.05\right)$. Este resultado indica un patrón de crecimiento similar en ambos años para esta variable, aunque con diferente tasa de crecimiento, como se corrobora con los parámetros obtenidos de las ecuaciones del modelo logístico: ANTE $_{1998}=55.47\left\{\mathrm{e}^{-0.07(\text { edad }-1.21)}+1\right\}^{-1}, \mathrm{R}^{2}=0.88$ y $\mathrm{ANTE}_{2001}=50.39\left\{\mathrm{e}^{-0.10}\right.$ (edad - 0.95) +1$\}^{-1}, R^{2}=0.92$

Con respecto al peso, los ejemplares del 2001 fueron significativamente mayores que los de 1998 los primeros 20 días, después estas diferencias dejaron de ser significativas en las categorías de edad 25 y 30 (Tabla 2, Fig. 3 c y d). Los primeros 20 días, la tasa

\begin{tabular}{llllll}
\hline \multirow{2}{*}{ Edad } & \multirow{2}{*}{$\begin{array}{l}\text { Hembras } \\
\text { Media } \pm \text { e.e. }\end{array}$} & $\begin{array}{l}\text { Machos } \\
\text { Media } \pm \text { e.e. }\end{array}$ & g.l. & $\mathbf{t}$ & $\mathbf{p}$ \\
\cline { 5 - 6 } & & & & & \\
\multicolumn{2}{l}{ Longitud del antebrazo } & & & & \\
1 & $26.74 \pm 0.55$ & $25.59 \pm 0.44$ & 35 & 1.50 & 0.12 \\
5 & $30.49 \pm 0.64$ & $29.23 \pm 0.61$ & 30 & 1.40 & 0.16 \\
10 & $35.37 \pm 0.60$ & $34.59 \pm 0.73$ & 31 & 0.82 & 0.41 \\
15 & $39.73 \pm 0.76$ & $39.72 \pm 0.65$ & 34 & 0.01 & 0.99 \\
20 & $43.87 \pm 0.57$ & $42.63 \pm 0.72$ & 25 & 1.36 & 0.18 \\
25 & $47.64 \pm 0.65$ & $44.96 \pm 1.05$ & 12 & 1.27 & 0.14 \\
30 & $50.90 \pm 1.33$ & $47.47 \pm 1.06$ & 4 & 1.90 & 0.12 \\
\hline
\end{tabular}

Peso

\begin{tabular}{lrrrrl}
1 & $5.25 \pm 0.16$ & $5.51 \pm 0.12$ & 35 & 1.23 & 0.22 \\
5 & $6.75 \pm 0.25$ & $6.73 \pm 0.21$ & 30 & 0.07 & 0.93 \\
10 & $7.95 \pm 0.25$ & $8.42 \pm 0.25$ & 31 & 1.29 & 0.20 \\
15 & $9.37 \pm 0.30$ & $9.99 \pm 0.29$ & 34 & 1.42 & 0.16 \\
20 & $11.46 \pm 0.33$ & $11.75 \pm 0.37$ & 25 & 0.57 & 0.57 \\
25 & $14.31 \pm 0.45$ & $13.06 \pm 0.65$ & 12 & 1.61 & 0.13 \\
30 & $15.65 \pm 0.25$ & $13.90 \pm 0.35$ & 4 & 1.64 & 0.07 \\
\hline
\end{tabular}

Longitud de la epífisis metacarpo-falange del $4^{\circ}$ dedo

\begin{tabular}{llllll}
1 & $4.60 \pm 0.08$ & $4.59 \pm 0.07$ & 35 & 0.02 & 0.97 \\
5 & $5.29 \pm 0.10$ & $5.22 \pm 0.09$ & 30 & 0.48 & 0.63 \\
10 & $5.75 \pm 0.10$ & $5.74 \pm 0.10$ & 31 & 0.11 & 0.90 \\
15 & $6.04 \pm 0.13$ & $6.31 \pm 0.09$ & 34 & 1.62 & 0.11 \\
20 & $5.89 \pm 0.12$ & $6.06 \pm 0.12$ & 25 & 0.94 & 0.35 \\
25 & $5.55 \pm 0.12$ & $6.04 \pm 0.24$ & 12 & 1.97 & 0.07 \\
30 & $4.75 \pm 0.36$ & $3.20 \pm 0.24$ & 4 & 2.50 & 0.06 \\
\hline
\end{tabular}

e.e. = error estándar, g.I. grados de libertad, $\mathrm{t}=$ valor de $t$ calculado, $\mathrm{p}=$ probabilidad a la que es significativo el valor de $t$. 
Tabla 1 Continúa...

\begin{tabular}{|c|c|c|c|c|c|}
\hline \multirow{2}{*}{ Edad } & \multirow{2}{*}{$\begin{array}{l}\text { Hembras } \\
\text { Media } \pm \text { e.e. }\end{array}$} & \multirow{2}{*}{$\begin{array}{l}\text { Machos } \\
\text { Media } \pm \text { e.e. }\end{array}$} & \multicolumn{3}{|r|}{2001} \\
\hline & & & g.l. & $t$ & $\mathbf{p}$ \\
\hline \multicolumn{6}{|c|}{ Longitud del antebrazo } \\
\hline 1 & $27.40 \pm 0.33$ & $26.69 \pm 0.31$ & 35 & 1.52 & 0.13 \\
\hline 5 & $32.68 \pm 049$ & $31.57 \pm 0.41$ & 22 & 1.71 & 0.10 \\
\hline 10 & $37.92 \pm 0.61$ & $36.82 \pm 0.84$ & 26 & 1.07 & 0.29 \\
\hline 15 & $42.41 \pm 0.51$ & $41.78 \pm 0.44$ & 33 & 0.92 & 0.36 \\
\hline 20 & $45.78 \pm 0.30$ & $44.40 \pm 0.69$ & 30 & 1.81 & 0.08 \\
\hline 25 & $47.79 \pm 0.68$ & $45.00 \pm 1.34$ & 12 & 1.85 & 0.08 \\
\hline 30 & $49.40 \pm 0.89$ & $46.60 \pm 2.57$ & 3 & 1.25 & 0.29 \\
\hline 35 & 52.49 & & & & \\
\hline \multicolumn{6}{|l|}{ Peso } \\
\hline 1 & $6.28 \pm 0.16$ & $6.55 \pm 0.18$ & 35 & 1.05 & 0.30 \\
\hline 5 & $8.29 \pm 0.17$ & $8.33 \pm 0.15$ & 22 & 0.17 & 0.86 \\
\hline 10 & $9.73 \pm 0.21$ & $9.61 \pm 0.27$ & 26 & 0.33 & 0.73 \\
\hline 15 & $11.40 \pm 0.24$ & $11.65 \pm 0.28$ & 33 & 1.35 & 0.18 \\
\hline 20 & $12.31 \pm 0.35$ & $12.53 \pm 0.33$ & 30 & 0.45 & 0.65 \\
\hline 25 & $12.92 \pm 0.75$ & $12.65 \pm 0.62$ & 12 & 0.27 & 0.78 \\
\hline 30 & $14.33 \pm 1.20$ & $13.25 \pm 1.25$ & 3 & 0.59 & 0.59 \\
\hline 35 & 17.50 & & & & \\
\hline \multicolumn{6}{|c|}{ Longitud de la epífisis metacarpo-falange del cuarto dedo } \\
\hline 1 & $3.69 \pm 0.06$ & $3.88 \pm 0.07$ & 35 & 1.83 & 0.07 \\
\hline 5 & $4.29 \pm 0.12$ & $4.28 \pm 0.06$ & 22 & 0.06 & 0.94 \\
\hline 10 & $4.80 \pm 0.12$ & $4.94 \pm 0.09$ & 26 & 0.90 & 0.37 \\
\hline $15^{*}$ & $4.56 \pm 0.09$ & $4.93 \pm 0.07$ & 33 & 1.68 & 0.008 \\
\hline $20^{*}$ & $4.30 \pm 0.06$ & $4.53 \pm 0.05$ & 30 & 2.70 & 0.01 \\
\hline 25 & $4.02 \pm 0.12$ & $4.19 \pm 0.14$ & 12 & 0.89 & 0.38 \\
\hline 30 & $3.76 \pm 0.09$ & $4.24 \pm 0.06$ & 3 & 1.72 & 0.06 \\
\hline 35 & 3.17 & & & & \\
\hline
\end{tabular}

de aumento del peso fue de 0.31 g/día en 1998 y de 0.30 g/día en el 2001, después disminuyó a 0.29 g/día en 1998 y a 0.14 g/día en el 2001. La comparación de las curvas de crecimiento entre años indica que durante los primeros 20 días éstas tuvieron comportamiento significativamente diferente (pendiente: $t_{(2) 317 \text { g.l. }}=2.25, P<0.05$; elevación: $\left.\mathrm{t}_{(2) 317 \text { g.l. }}=3.40, P<0.05\right)$, mientras que del día 20 al 30 las diferencias dejaron de ser significativas (pendiente: $\mathrm{t}_{(2) 94 \text { g.l. }}=0.44, P>0.05$; elevación: $\mathrm{t}_{(2) 94 \text { g.l. }=} 1.23, \mathrm{p}>0.05$ ). Las ecuaciones derivadas del modelo logístico son: $\mathrm{PESO}_{1998}=22.31\left\{\mathrm{e}^{-0.06(\mathrm{t}-3.29)}+1\right\}^{-1}, \mathrm{R}^{2}$ $=0.99$ y $\mathrm{PESO}_{2001}=14.69\left\{\mathrm{e}^{-0.10(\mathrm{t}-1.37)}+1\right\}^{-1}, \mathrm{R}^{2}=0.82$, resultados que indican un patrón $\mathrm{y}$ tasa de crecimiento diferente en cada año.

La longitud de la epífisis cartilaginosa del cuarto dedo fue significativamente mayor en todas las categorías de edad de 1998 en comparación con 2001 (Tabla 2), lo que sugiere 
una mayor tasa de osificación en el segundo año. Ésta variable aumentó constantemente hasta el quinceavo día en 1998 y al décimo día en el 2001, con una tasa de crecimiento de $0.10 \mathrm{~mm} /$ día en ambos años. Mientras que la osificación fue de 0.05 mm/día en 1998 y 0.04 mm/día en el 2001 (Fig. 3 e y f). La comparación de las curvas de crecimiento entre años indica que éstas fueron similares en la primera fase de crecimiento (pendiente: $\mathrm{t}_{(2) 223 \text { g.l. }}=1.58, P>0.05$; elevación: $\left.\mathrm{t}_{(2) 222 \text { g.l. }}=0.44, P>0.05\right)$, pero significativamente diferentes en la segunda fase (pendiente: $\mathrm{t}_{(2) 195 \text { g.l. }}=3.87, P<0.001$; elevación: $\mathrm{t}_{(2) 194 \text { g.l. }}=$ 2.83, $P<0.01$ ), resultado que muestra un patrón de crecimiento diferente en cada año.

De varios modelos polinomiales ensayados, un modelo cuadrático fue el que mejor se ajustó a los datos:

$$
\begin{aligned}
& \mathrm{EPI}_{1998}=\left((4.3732+(0.2093) *(\mathrm{edad})) /\left(1+(0.00107) *(\mathrm{edad})^{2}\right)\right), \mathrm{R}^{2}=0.61 \mathrm{y} \\
& \mathrm{EPI}_{2001}=\left((3.5720+(0.2057) *(\text { edad })) /\left(1+(0.00179) *(\mathrm{edad})^{2}\right)\right), \mathrm{R}^{2}=0.57
\end{aligned}
$$

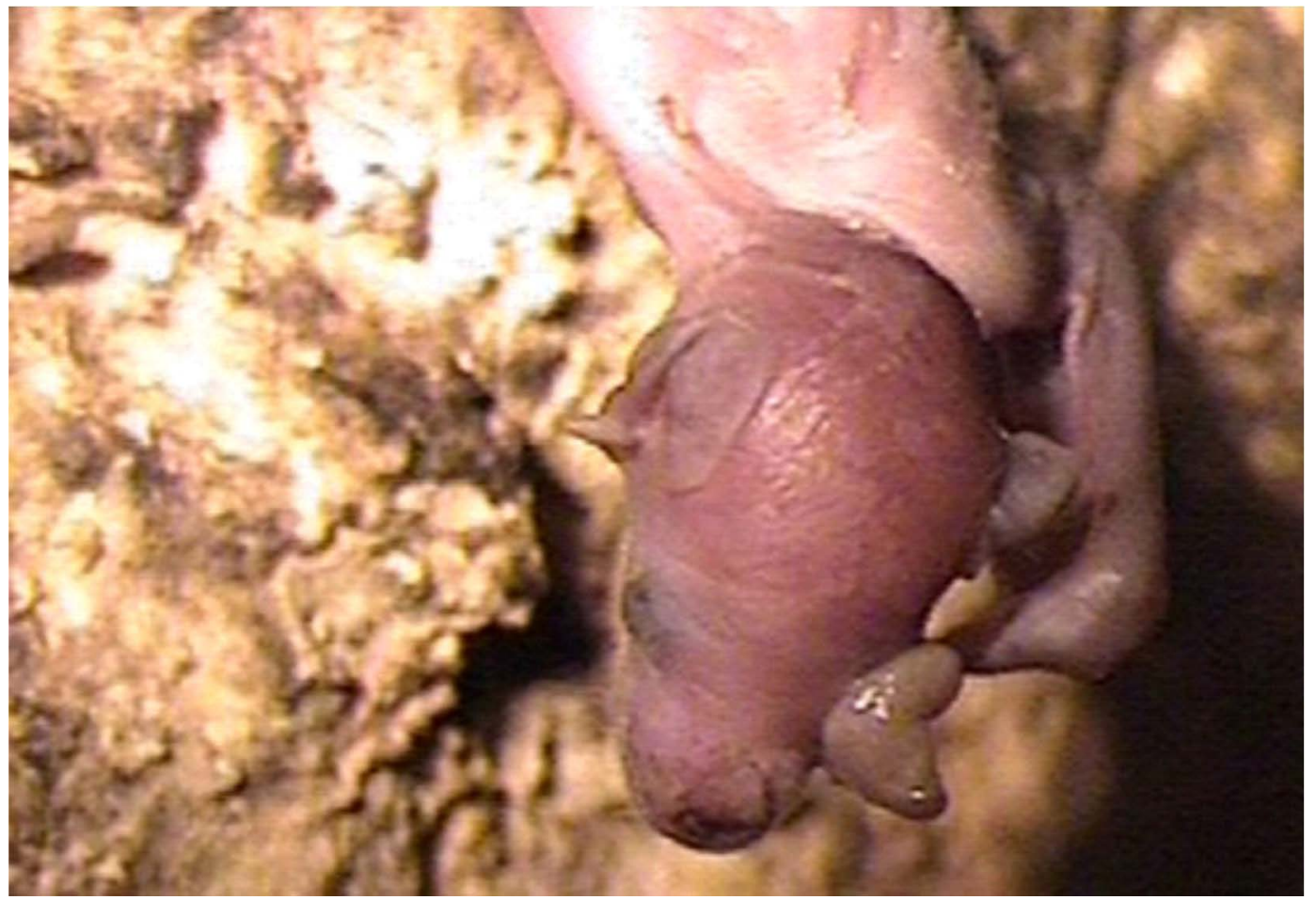

Figura 2. Neonato de Leptonycteris yerbabuenae en la cueva de Los Laguitos, Chiapas, México. Se observa la piel rosada y desnuda, las orejas plegadas a la cabeza, los ojos cerrados y la placenta fresca a un lado del rostro.

Desarrollo del vuelo. En los dos años de estudio todos los recién nacidos y hasta los 10 días de edad fueron clasificados como no volantones, así como dos ejemplares de 1998 que siempre se rehusaron a volar, por lo cual fueron clasificados como no volantones hasta los 30 días de edad (Tabla 3). Entre los 15 y 25 días de edad la mayoría de los ejemplares fueron semivolantones, 28 en 1998 y 29 en 2001. El vuelo sostenido inició a los 15 días en 2001 (dos ejemplares) y a los 20 días de edad en 1998 (tres ejemplares). A los 30 días todos los individuos capturados fueron volantones, a excepción de los ya mencionados.

En los individuos que lograron el vuelo sostenido por primera vez, la longitud del antebrazo representó el $83.79 \%$ del adulto en 1998 y el $84.16 \%$ en el 2001, mientras que la relación del peso del volantón respecto al adulto fue de 49.54\% para 1998 y de $54.59 \%$ para el 2001. La longitud de la epífisis de los volantones fue de $5.78 \mathrm{~mm}$ para los de 1998 y de 4.37 mm en 2001. Cuando se inició el vuelo sostenido no se observó 
una pérdida de peso en la mayoría de los individuos, aunque el ritmo de crecimiento disminuyó durante este período (Fig. 3). A los 30 días todos los volantones recapturados habían alcanzado el $88.28 \%$ del antebrazo del adulto postparto en 1998 y el $88.02 \%$ en 2001, mientras que la relación del peso fue de 55.50\% en 1998 y 58.25\% en 2001 .

Figura 3.- Cambios debidos a la edad en la longitud del antebrazo (a y b), peso (c y d) y longitud de la epífisis cartilaginosa metacarpofalange del cuarto dedo (e y f) del murciélago Leptonycteris yerbabuenae en 1998 y 2001, con sus respectivas curvas y líneas de predicción de 95\% de confianza, derivadas del modelo logístico para las dos primeras variables y de un modelo polinomial cuadrático para la tercera variable.
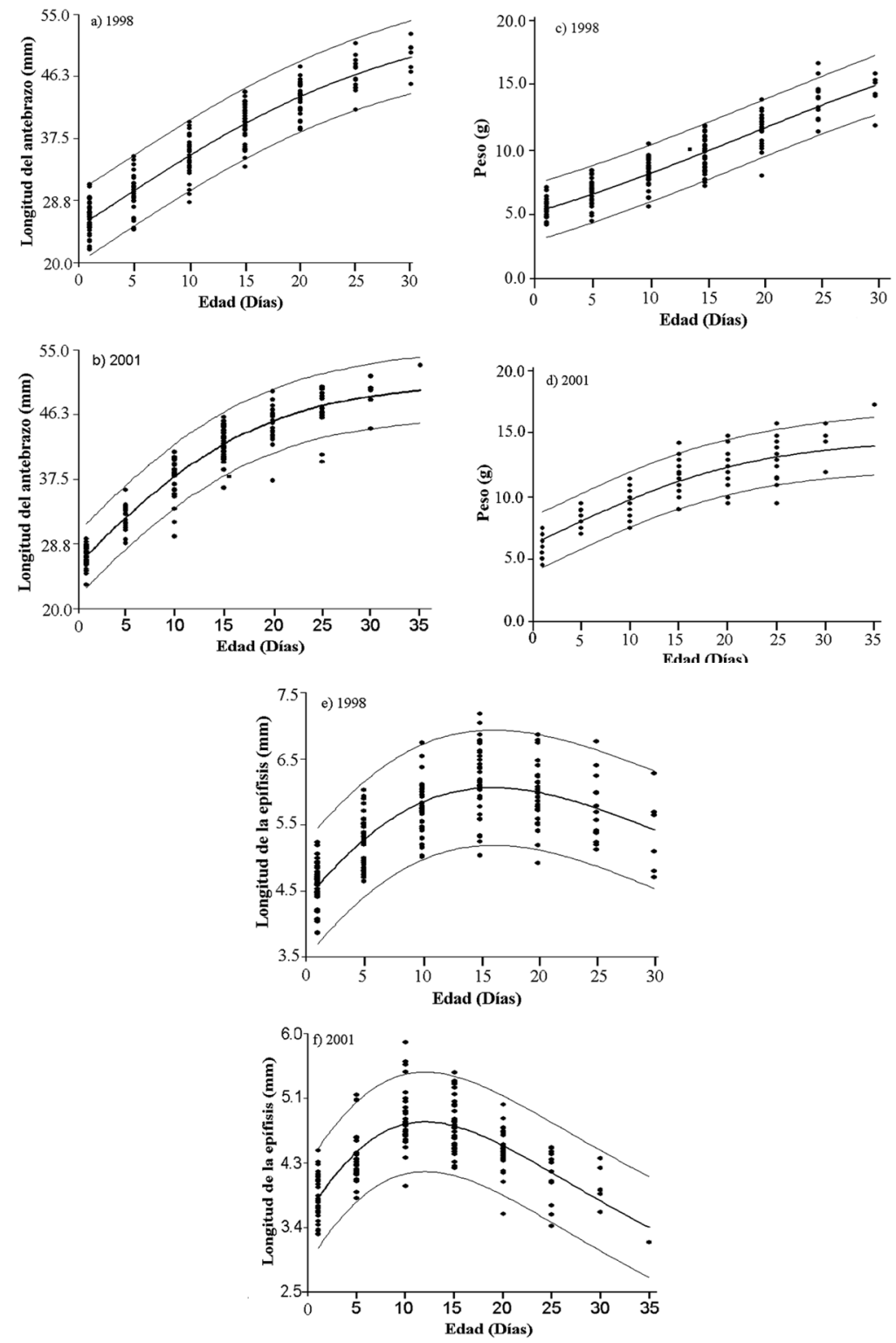

\section{Discusión}

En contraste con otras especies de murciélagos filostómidos como Artibeus jamaicensis, Carollia perspicillata o Glossophaga soricina que nacen cubiertos de pelo y con los ojos abiertos (Kleiman y Davis 1979), las crías de Leptonycteris yerbabuenae de Chiapas nacieron desnudas, con los ojos y el meato auditivo cerrados y con las orejas plegadas.

Nuestras observaciones contrastan con el reporte de Gould (1975), quien observó bajo condiciones de laboratorio, que hembras de $L$. yerbabuenae provenientes de Carbo, Sonora, dieron a luz a crías cubiertas de pelo y con el meato auditivo abierto. Estas diferencias parecen reflejar la variabilidad de la especie, que al parecer está estructurada 
por dos grupos que poseen comportamientos diferentes. Por ejemplo, se sabe que las poblaciones de $L$. yerbabuenae del norte de México se reproducen durante la primavera y se comportan como migratorias latitudinales, mientras que las del centro y sureste se reproducen en otoño e invierno y son residentes o migratorias locales (Wilkinson y Fleming 1996; Álvarez et al. 1999; Rojas-Martínez et al. 1999; Galindo et al. 2004).

\begin{tabular}{|c|c|c|c|c|c|c|c|c|c|}
\hline Edad & $\mathrm{n}$ & Media \pm e.e. & Intervalo & $\mathrm{n}$ & Media \pm e.e. & Intervalo & g.l. & $\mathrm{t}$ & $\mathrm{P}$ \\
\hline \multicolumn{10}{|c|}{ Longitud del antebrazo } \\
\hline 1 & 37 & $26.21 \pm 0.37$ & $21.87-31.00$ & 37 & $27.02 \pm 0.23$ & $23.14-29.25$ & 72 & 1.83 & 0.07 \\
\hline $5^{*}$ & 32 & $29.90 \pm 0.45$ & $24.67-34.96$ & 24 & $32.12 \pm 0.33$ & $28.71-35.87$ & 54 & 3.72 & 0.0004 \\
\hline $10^{*}$ & 33 & $35.01 \pm 0.46$ & $28.50-39.80$ & 28 & $37.41 \pm 0.51$ & $29.53-40.91$ & 59 & 3.44 & 0.001 \\
\hline $15^{*}$ & 36 & $39.73 \pm 0.46$ & $33.50-44.13$ & 35 & $42.09 \pm 0.33$ & $36.15-45.52$ & 69 & 4.09 & 0.0001 \\
\hline $20^{*}$ & 27 & $43.37 \pm 0.46$ & $38.80-47.61$ & 32 & $45.09 \pm 0.39$ & $37.04-49.02$ & 57 & 2.85 & 0.005 \\
\hline 25 & 14 & $46.68 \pm 0.64$ & $41.58-50.91$ & 14 & $46.39 \pm 0.82$ & $39.57-49.56$ & 26 & 0.27 & 0.78 \\
\hline 30 & 6 & $48.61 \pm 1.04$ & $45.17-52.23$ & 5 & $48.28 \pm 1.17$ & $44.03-50.95$ & 9 & 0.21 & 0.83 \\
\hline 35 & & & & 1 & 52.49 & & & & \\
\hline \multicolumn{10}{|l|}{ Peso } \\
\hline $1 *$ & 37 & $5.37 \pm 0.10$ & $4.20-7.10$ & 37 & $6.42 \pm 0.12$ & $4.50-7.50$ & 72 & 6.50 & 0.0000 \\
\hline $5^{*}$ & 32 & $6.74 \pm 0.16$ & $4.50-8.40$ & 24 & $8.31 \pm 0.11$ & $7.00-9.50$ & 54 & 7.31 & 0.0000 \\
\hline $10^{*}$ & 33 & $8.16 \pm 0.18$ & $5.60-10.50$ & 28 & $9.67 \pm 0.17$ & $7.50-11.50$ & 59 & 5.91 & 0.0000 \\
\hline $15^{*}$ & 36 & $9.66 \pm 0.21$ & $7.20-11.90$ & 35 & $11.40 \pm 0.19$ & $9.00-14.40$ & 69 & 5.98 & 0.0000 \\
\hline $20^{*}$ & 27 & $11.58 \pm 0.24$ & $8.00-13.90$ & 32 & $12.42 \pm 0.23$ & $9.50-15.00$ & 57 & 2.43 & 0.01 \\
\hline 25 & 14 & $13.86 \pm 0.39$ & $11.40-16.70$ & 14 & $12.79 \pm 0.47$ & $9.50-16.00$ & 26 & 1.74 & 0.09 \\
\hline 30 & 6 & $14.48 \pm 0.58$ & $11.90-15.90$ & 5 & $13.90 \pm 0.81$ & $12.00-16.00$ & 9 & 0.59 & 0.56 \\
\hline 35 & & & & 1 & 17.50 & & & & \\
\hline \multicolumn{10}{|c|}{ Longitud de la epífisis metacarpo-falange del cuarto dedo } \\
\hline $1^{*}$ & 37 & $4.59 \pm 0.05$ & $3.87-5.24$ & 37 & $3.79 \pm 0.05$ & $3.28-4.42$ & 72 & 10.82 & 0.0000 \\
\hline $5^{*}$ & 32 & $5.26 \pm 0.06$ & $4.65-6.03$ & 24 & $4.29 \pm 0.06$ & $3.77-5.17$ & 54 & 9.74 & 0.0000 \\
\hline $10^{*}$ & 33 & $5.74 \pm 0.07$ & $5.02-6.74$ & 28 & $4.87 \pm 0.07$ & $3.93-5.88$ & 59 & 8.19 & 0.0000 \\
\hline $15^{*}$ & 36 & $6.17 \pm 0.08$ & 5.04-7.18 & 35 & $4.75 \pm 0.05$ & $4.18-5.47$ & 69 & 13.46 & 0.0000 \\
\hline $20 *$ & 27 & $5.96 \pm 0.08$ & $4.92-6.86$ & 32 & $4.42 \pm 0.04$ & $3.56-5.04$ & 57 & 16.03 & 0.0000 \\
\hline $25^{*}$ & 14 & $5.72 \pm 0.13$ & $5.13-6.76$ & 14 & $4.11 \pm 0.09$ & $3.39-4.46$ & 26 & 10.02 & 0.0000 \\
\hline $30 *$ & 6 & $5.37 \pm 0.24$ & $4.71-6.28$ & 5 & $3.95 \pm 0.13$ & $3.58-4.31$ & 9 & 4.74 & 0.001 \\
\hline 35 & & & & 1 & 3.17 & & & & \\
\hline
\end{tabular}

Tabla 2. Variación interanual. Se presentan los estadísticos paramétricos de tres variables morfométricas y los resultados de una prueba de $t$ de Student, usada para evaluar las diferencias en los juveniles de Leptonycteris yerbabuenae en la cueva de "Los Laguitos", Chiapas. El * indica las categorías de edad en donde las diferencias entre años fueron significativas a una $\mathrm{P} \leq 0.05$.

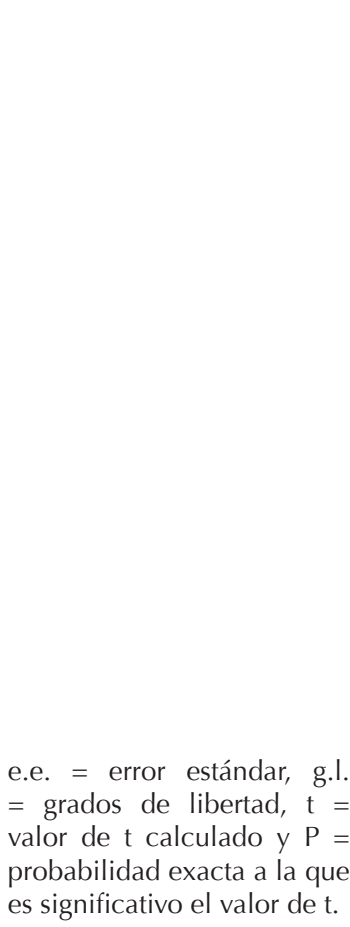

Aunque, Guevara-Carrizales et al. (2010) consideran que las poblaciones de Baja California posiblemente sean residentes en el estado, sin embargo sus observaciones no son concluyentes.

Los neonatos de 1998 nacieron con menos peso y con una epífisis cartilaginosa más grande que los de 2001. Diferentes factores pueden influir sobre el crecimiento y el desarrollo del feto, como son las condiciones ambientales (temperatura y HR) del refugio, así como las del medio externo (Kunz 1982 y 1987; Kunz y Hood 2000). En el caso del medio externo la precipitación afecta en diferente grado la disponibilidad 
de alimento para los murciélagos (Lobo et al. 2003; Borchert et al. 2004). Se sabe que los cambios de temperatura y HR, ya sea durante la gestación o la lactancia, Ilegan a influir sobre la tasa de crecimiento de los murciélagos; por lo que una disminución en la temperatura del refugio, retrasa la tasa del crecimiento y desarrollo del feto o de la cría y en especies que no entran en torpor, el costo de la termorregulación aumenta. En cambio el aumento de la temperatura acelera el metabolismo y consecuentemente la tasa de crecimiento (Tuttle y Stevenson 1977; Hoying y Kunz 1998; Speakman y Thomas 2003). Por lo tanto es posible que las condiciones ambientales que prevalecieron en ambos años, tanto en el medio externo (en 1998 la precipitación media fue $247 \mathrm{~mm}$ mayor, mientras que la temperatura media fue $2.9{ }^{\circ} \mathrm{C}$ menor respecto a 2001) como en el refugio (en 1998 la HR fue 5.19\% mayor, mientras que la temperatura media fue de $0.75{ }^{\circ} \mathrm{C}$ menor respecto a 2001) expliquen porque los neonatos de 2001 nacieron más pesados y con mayor osificación en la epífisis que los de 1998. Estos resultados contrastan con lo reportado para Phyllostomus hastatus, en donde Stern y Kunz (1998) no pudieron relacionar ninguna variable climática con las diferencias en la talla de los neonatos de años diferentes.

La Cueva de los Laguitos registró en los dos años de estudio una temperatura $>32$ ${ }^{\circ} \mathrm{C}$ y una HR $>90 \%$, condiciones consideradas idóneas para la reproducción de estos murciélagos (Silva-Taboada 1979; Betts 1997; Heideman 2000; Speakman y Thomas 2003), pues se encuentra dentro de la zona termoneutral $\left(>30.5^{\circ} \mathrm{C}\right)$. Esta temperatura ha sido identificada para otros murciélagos glosofagínos, entre los que se encuentra $L$. curasoae (Arends et al. 1995), especie hermana de L. yerbabuenae. Asimismo, Arends et al. (1995) reportan que $L$. curasoae, en Sudamérica, utiliza como sitio de maternidad cuevas con temperaturas que varían de 33 a $34.2{ }^{\circ} \mathrm{C}$, valores similares a los encontrados en la cueva de Los Laguitos durante el periodo de estudio. Si L. yerbabuenae posee una zona termoneutral similar a la de L. curasoae, entonces, los valores de temperatura y HR que prevalecieron en Los Laguitos fueron importantes para el crecimiento de sus crías, sobre todo si se considera que estas nacen desnudas y con baja capacidad termorreguladora.

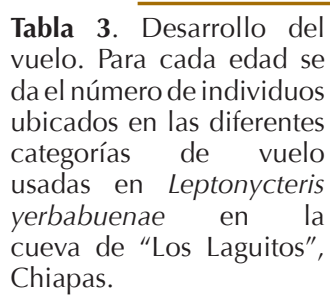

Tabla 3. Desarrollo del vuelo. Para cada edad se da el número de individuos ubicados en las diferentes categorías de vuelo usadas en Leptonycteris yerbabuenae en la cueva de "Los Laguitos", Chiapas.

\begin{tabular}{llllllll}
\hline \multirow{2}{*}{ Categoría de vuelo } & \multicolumn{2}{l}{ Edad } & & & & \\
\cline { 2 - 7 } & 1 & 5 & 10 & 15 & 20 & 25 & 30 \\
\hline 1998 & & & & & & & \\
No volantón & 37 & 32 & 33 & 18 & 17 & 2 & 2 \\
Semivolantón & & & & 18 & 7 & 4 & \\
Volantón & & & & & 3 & 8 & 4 \\
\hline 2001 & \multirow{2}{*}{24} & 28 & 21 & 10 & & \\
No volantón & 37 & & & 12 & 11 & 5 & \\
Semivolantón & & & & 2 & 11 & 9 & 5 \\
Volantón & & & & & & & \\
\hline
\end{tabular}

Otros factores que influyen sobre el crecimiento de los neonatos son: la talla al nacer, el sexo y el tamaño de la camada (Basset 1984, Stern y Kunz 1998). El tamaño de los neonatos parece haber sido otro factor importante durante la etapa de crecimiento. Nuestros resultados muestran que los ejemplares de 2001, que fueron los más pesados y con la epífisis más osificada, mantuvieron o aumentaron sus diferencias (en el caso de la longitud del antebrazo) con respecto a los de 1998 durante los primeros 20 
días. Después de éste tiempo las diferencias disminuyeron. La influencia del sexo es notable en las especies de murciélagos donde existe dimorfismo sexual, debido a que la tasa de crecimiento es más acelerada en el sexo de talla mayor, tal como sucede en los machos de P. hastatus o en las hembras de Lasiurus cinereus (Stern y Kunz 1998; Koehler y Barclay 2000). Sin embargo, en L. yerbabuenae no existe dimorfismo sexual entre adultos (Arita y Humphrey 1988), neonatos o juveniles. Por lo tanto, era de esperar que no hubiese diferencias en la tasa y el patrón del crecimiento entre los sexos de esta especie.

El tamaño relativo de la longitud del antebrazo (47.60 - 49.26\%) en los neonatos de L. yerbabuenae, en relación con los adultos es grande, en comparación con algunos vespertiliónidos, hiposidéridos, tiroptéridos y megadermátidos que produjeron también una sola cría (Liu et al. 2009; Lin et al. 2010; Chaverri y Vonhof 2011). En relación con otros filostómidos que paren una cría, se encontró que la longitud del antebrazo de $L$. yerbabuenae, fue mayor en comparación con $P$. hastatus (42\%) pero menor que Artibeus watsoni (54 a 59\%). Mientras que en el peso, los neonatos de L. yerbabuenae (20.58 - 26.90\%) está cercano al promedio de 22.3\% (12 - 43\%) que Kurta y Kunz (1987) obtuvieron para los murciélagos estudiados hasta esa fecha. Asimismo, su peso relativo fue similar al de $P$. hastatus (20.7\%), pero menor en comparación con $A$. watsoni (30 - 34\%), Hipposideros pomona (39.7\%), Myotis macrodactylus (39.6\%) y Thryroptera tricolor (26.02 - 31.43\%; Stern y Kunz 1998; Chaverri y Kunz 2006; Liu et al. 2009; Lin et al. 2010; Chaverri y Vonhof 2011).

Al igual que en otras especies de murciélagos, el peso y la longitud del antebrazo de $L$. yerbabuenae experimentaron aumentos lineales en el periodo previo al vuelo. Una vez que empezaron a volar, la tasa de crecimiento disminuyó. Los individuos del 2001, en comparación con los de 1998, tuvieron una velocidad de crecimiento mayor durante los primeros 20 días, posiblemente como consecuencia de la temperatura mayor que hubo en el 2001. La epífisis cartilaginosa del cuarto dedo experimentó cambios similares a los reportados para otras especies; esto es, primero hubo un crecimiento del tejido cartilaginoso y después empezó a calcificarse, justo antes de que los individuos empezaran a volar (Burnett y Kunz 1982; Kunz y Anthony 1982; Stern y Kunz 1998). Los individuos del 2001 tuvieron una epífisis más osificada, la cual empezó a cerrarse cinco días antes que en los de 1998, razón por la que tal vez el vuelo inició cinco días antes en los ejemplares del 2001.

La longitud de la epífisis cartilaginosa ha sido frecuentemente sugerida como criterio para estimar la edad precisa en murciélagos jóvenes con fecha de nacimiento desconocida (Kunz y Hood 2000; Liu et al., 2009; Lin et al. 2010). Sin embargo, los resultados obtenidos en el presente estudio, así como en otros trabajos (Hoying y Kunz 1998), muestran que el uso de tales modelos predictivos no puede ser aplicado indiscriminadamente, debido a que las condiciones del ambiente son siempre cambiantes y aun pequeñas diferencias pueden modificar la tasa de crecimiento. Por lo tanto, la aplicación de estos modelos para estimar la edad de individuos silvestres debe utilizarse con las reservas pertinentes.

Durante el aprendizaje del vuelo, los jóvenes de algunas especies de murciélagos (Myotis velifer, M. lucifugus, Pipistrellus pipistrellus y P. subflavus) pierden peso, razón por la que se considera esta etapa como un periodo fisiológicamente estresante 
para ellos (Kunz 1987; Hoying y Kunz 1998). Sin embargo, en otras especies como Antrozous pallidus, Eptesicus fuscus y Rhinolophus cornutus no ocurre tal pérdida. Estas diferencias posiblemente estén relacionadas con la obtención de energía, que en estas últimas especies no es un problema, ya que existe un cuidado materno prolongado, fenómeno que no ocurre en las especies de Myotis estudiadas (Kunz 1987). En el caso de L. yerbabuenae no existe una pérdida de peso durante el inicio del vuelo, aunque sí una disminución en la tasa de crecimiento que se considera normal, ya que el individuo ha alcanzado, al menos en el antebrazo, dimensiones cercanas a las del adulto. Además, el peso es afectado por múltiples variables difíciles de controlar, como son la disponibilidad de alimento, la capacidad de la madre para procesar cada noche la calidad nutritiva de la leche, la salud de la madre y desde luego la capacidad metabólica del joven, entre otros (Kunz 1987).

El vuelo exige que los juveniles tengan un esqueleto osificado, que resista la tensión a la que los huesos están sometidos durante el aleteo, así como desarrollar una buena coordinación neuromuscular y haber desarrollado la ecolocalización (Kunz 1987). Si alguna de estas características no se logran después del destete, los juveniles están condenados a morir, porque la supervivencia depende del vuelo. En L. yerbabuenae el vuelo inició entre los 15-20 días de edad y está dentro del rango de 2.5 a 4 semanas que está reportado en otros filostómidos (Kleiman y Davis 1979) y vespertiliónidos (Hoying y Kunz 1998; Krochmal y Sparks 2007; Liu et al. 2009). El inicio del vuelo sostenido apareció cinco días antes en los juveniles del 2001 en comparación con 1998, y consideramos es consecuencia de la mayor osificación en la epífisis, el mayor peso y longitud de antebrazo que siempre mantuvieron durante su trayectoria de crecimiento. Un caso similar ocurrió en Myotis grisescens, especie en la cual el inicio del vuelo se presentó nueve días antes en una colonia con una temperatura de $16.4{ }^{\circ} \mathrm{C}$ en comparación con otra de $13.9{ }^{\circ} \mathrm{C}$ (Tuttle 1975).

A los 30 días de edad todos los individuos recapturados de L. yerbabuenae eran voladores, excepto dos individuos que no lo hicieron; aunque cinco días después no los volvimos a encontrar. En los individuos de 30 días de edad la talla relativa del antebrazo en relación con el adulto fue de 88.02 a $88.28 \%$, algo menor al reportado para H. larvatus $(92.44 \%)$, T. tricolor $(95 \%)$, M. macrodactylus $(97.1 \%)$ y A. watsoni $(100 \%)$ de la misma edad. Asimismo, respecto al peso relativo de los juveniles de 30 días de edad de $L$. yerbabuenae (55.50 - 58.25\%), fue menor al reportado para $H$. larvatus (61.42\%), Pipistrellus subflavus (68 - 80\%), T. tricolor (77\%), A. watsoni $(80 \%)$ y M. macrodactylus (94.7\%; Hoying y Kunz 1998; Chaverri y Kunz 2006; Liu et al. 2009; Lin et al. 2010; Chaverri y Vonhof 2011). Con relación a lo anterior, observamos que el peso de los jóvenes de 30 días de L. yerbabuenae son menores a los de la mayoría de las especies.

Desconocemos que factores estén relacionados con este hecho, pero es posible que la alta capacidad de vuelo de los adultos de $L$. yerbabuenae, que deben suspender el vuelo y maniobrar para acercarse a las flores durante su alimentación, exige que inicien el vuelo lo antes posible (Horner et al. 1998). Los valores de la constante de crecimiento derivados del modelo logístico obtenidos para la longitud del antebrazo $(0.07-0.10)$ y peso $(0.06-0.10)$ de $L$. yerbabuenae, son comparables a los reportados para otros filostómidos neotropicales como Artibeus jamaicensis (0.08 
y 0.04 respectivamente), Carollia perspicillata (0.01 y 0.10) y P. hastatus (0.07 y 0.06 ; Kunz y Hood 2000) y ligeramente menores en comparación con los reportados para los vespertilionidos Pipistrellus subflavus (0.11 - 0.13 y 0.13), Eptesicus fuscus (0.13), Myotis macrodactylus (0.14) y Myotis sp. (0.12 - 0.27; Hoying y Kunz 1998; Kunz y Hood 2000; Liu et al. 2009). Es posible que estas diferencias reflejen la distancia filogenética de los grupos comparados, además del efecto del ambiente. Los vespertiliónidos citados, se distribuyen en las regiones templadas, y son habitantes de ambientes con una estación benigna corta y por lo tanto, las crías deben acelerar su crecimiento para poder migrar o hibernar (Heideman 2000), en comparación con los filostómidos que viven en ambientes tropicales estables.

Conocer la estrategia de crecimiento de cualquier especie es importante, pero en el caso de una especie amenazada, como es el caso de $L$. yerbabuenae es prioritario, debido a que este conocimiento proporciona los elementos para entender las adaptaciones de la especie y su dependencia a las cavernas como refugios de maternidad, ya que sus crías nacen en menor estado de desarrollo, en comparación con otros filostómidos como A. jamaicensis y C. perspicillata. Es necesario establecer un programa de protección y manejo de la especie y de los sitios que utiliza como refugios diurnos y en especial de las cavernas, que hasta el momento se sabe utiliza como sitios de reproducción.

A los dos revisores anónimos cuyos comentarios y observaciones permitieron mejorar sustancialmente el texto original. A M. I. Verona Trejo por su apoyo en la redacción final del manuscrito. A nuestros compañeros A. X. Hernández Cruz, E. García Hernández y A. Torres Jiménez por su apoyo en el trabajo de campo. M. Blas y M. Pérez Gutiérrez apoyaron incondicionalmente nuestra estancia en Tuxtla Gutiérrez.

Atcorn, S. M. 1962. Pollination requirements of the organ pipe cactus. Cactus Literatura citada Succulentus Journal 34:134-138.

Alcorn, S. M., S. E. McGregor, y G. Colin. 1961. Pollination of saguaro by doves, nectar feeding bats and honey bees. Science 133:1549-1555.

Álvarez, T., y L. González-Quintero. 1969. Análisis polínico del contenido gástrico de murciélagos Glossophaginae de México. Anales de la Escuela Nacional de Ciencias Biológicas 18:137-165.

Álvarez, T., N. Sánchez-Casas, y J. A. Villalpando. 1999. Registro de los movimientos de Leptonycteris yerbabuenae en el centro de México. Anales de la Escuela Nacional de Ciencias Biológicas 45:9-15.

Arends, A., F. J. Bonaccorso, y M. Genoud. 1995. Basal rates of metabolism of nectarivorous bats (Phyllostomidae) from a semiarid thorn forest in Venezuela. Journal of Mammalogy 76:947-956.

Arita, H. T. 1993. Conservation biology of Mexican cave bats. Journal of Mammalogy 74:693-702.

Arita, H. T., y S. R. Humphrey. 1988. Revisión Taxonómica de los murciélagos magueyeros del género Leptonycteris (Chiroptera: Phyllostomidae). Acta Zoológica Mexicana (n. s.) 29:1-60. 
Basset, J. F. 1984. Litter size and postnatal growth rate in the pallid bat, Antrozous pallidus. Journal of Mammalogy 65:317-319.

Betts, B. J. 1997. Microclimate in Hell's Canyon mines used by maternity colonies of Myotis yumanensis. Journal of Mammalogy 78:1240-1250.

Borchert, R., S. A. Meyer, R. S. Felger, y L. Porter-Bolland. 2004. Environmental control of flowering periodicity in Costa Rican and Mexican tropical dry forests. Global Ecology and Biogeography 13:409-425.

Burnett, C. D., y T. H. Kunz. 1982. Growth rates and age estimation in Eptesicus fuscus and comparison with Myotis lucifugus. Journal of Mammalogy 63:33-41.

Cardoso, C. M. D. 1979. El clima de Chiapas y Tabasco. Universidad Autónoma de México. Ciudad de México, México.

Chaverri, G., y T. H. Kunz. 2006. Reproductive biology and postnatal development in the tentmaking bat Artibeus watsoni (Chiroptera: Phyllostomidae). Journal of Zoology 270:650-656.

Chaverri, G., y M. J. Vonhof. 2011. Reproduction and growth in a Neotropical insectivorous bat. Acta Chiropterologica 13:147-155.

Cockrum, E. L., y Y. Petryszyn. 1991. The long-nosed bat, Leptonycteris: an endangered species in the southwest? Occasional Papers of the Museum, Texas Tech University 142:1-32.

De la Cruz, J. 1992. Bioecología de las cuevas de calor. Mundos Subterráneos 3:7-21.

Fleming, T. H., R. A. Nuñez, y S. L. L. Da Silveira. 1993. Seasonal changes in the diets of migrant and non-migrant nectarivorous bats as revealed by carbon stable isotope analysis. Oecologia 94:72-75.

Galindo-G., C., A. Sánchez-Q., R. H. Quijano, y L. G. Herrera-M. 2004. Population dynamics of a resident colony of Leptonycteris curasoae (Chiroptera: Phyllostomydae) in central Mexico. Biotropica 36:382-391.

Guevara-Carrizales, A. A., R. Martínez-Gallardo, y A. Moreno-Valdez. 2010. Primer registro de una colonia de Leptonycteris curasoae (Chiroptera: Phyllostomidae) en Baja California, México. Revista Mexicana de Biodiversidad 81:583-585.

GoutD, E. 1975. Neonatal vocalizations in bats of eight genera. Journal of Mammalogy 56:15-29.

Heideman, P. D. 2000. Environmental regulation of reproduction. pp. 469-499. In Reproductive Biology of Bats (Crichton, E. G., y P. H. Krutzsch, eds.). Academic Press. London, Reino Unido.

Hintze, J. L. 2007. Number Cruncher Statistical System (NCSS). Ver. 01.1.21. Kaysville, EE.UU.

Horner, M. A., T. H. Fleming, y C. T. Sahley. 1998. Foraging behaviour and energetics of a nectar-feeding bat, Leptonycteris curasoae (Chiroptera: Phyllostomidae). Journal of Zoology, London 244:575-586.

Hoying, K. M., y T. H. Kunz. 1998. Variation in size at birth and post-natal growth in the insectivorous bat Pipistrellus subflavus (Chiroptera: Vespertilionidae). Journal of Zoology 245:15-27. 
Kıeiman, D. G., y T. M. Davis. 1979. Ontogeny and maternal care. Pp. 387-402. in Biology of bats of the New World family Phyllostomidae. Part III (Baker, R. J., J. K. Jones Jr., y D. C. Carter, eds.). Special Publications of the Museum, Texas Tech University, 16.

Koehler, C., y R. M. R. Barclay. 2000. Post-natal growth nd breeding biology of the hoary bat (Lasiurus cinereus). Journal of Mammalogy 81:234-244.

Krochmal, A. R., y D. W. Sparks. 2007. Timing of birth and estimation of age of juvenile Myotis septentrionalis and Myotis lucifugus in west-central Indiana. Journal of Mammalogy 88:649-656.

Kunz, T. H. 1982. Roosting ecology of bats. Pp. 1-55 in Ecology of Bats (Kunz, T. H., ed.). Plenum Press. New York, EE.UU.

Kunz, T. H. 1987. Post-natal growth and energetics of suckling bats. Pp. 395-420 in Recent advances in the study of bats (Fenton, M. B., P. Racey, y J. M. V. Rayner, eds.). Cambridge University Press. Cambridge, EE.UU.

Kunz, T. H., y E. L. P. Anthony. 1982. Age estimation and post-natal growth in the bat Myotis lucifugus. Journal of Mammalogy 63:23-32.

Kunz T. H., y W. R. Hood. 2000. Parental care and postnatal growth in the chiroptera. pp. 415-468 en Reproductive Biology of Bats (Crichton, E. G., y P. H. Krutzsch, eds.). Academic Press. London, Reino Unido.

Kurta, A., y T. H. Kunz. 1987. Size of bats at birth and maternal investment during pregnancy. Symposium of the Zoological Society of London 57:79-106.

Lin, A. Q., L. R. JiN, Y. Liu, K. P. Sun, Y J. FenG. 2010. Postnatal growth and age estimation in horsfield's leaf-nosed bat Hipposideros larvatus. Zoological Studies 49:789796.

Liv, Y., L. R. Jin, W. Metzner, Y J. Feng. 2009. Postnatal growth and age estimation in bigfooted myotis, Myotis macrodactylus. Acta Chiropterologica 11:105-111.

Lobo, J. A., M. Quesada, K. E. Stoner, E. J. Fuchs, Y. Herrería-Diego, J. Rojas, y G. Saborío. 2003. Factors affecting phenological patterns of bombacaceous trees in seasonal forests in Costa Rica and Mexico. American Journal of Botany 90:1054-1063.

Martínez-Coronel, M., E. García, C. Müdespacher-Zihel, y A. Torres. 2010. El microclima de la cueva de "Los Laguitos" y su relación con los murciélagos. Mundos Subterráneos 21:74-89.

Miranda, F. 1998. La vegetación de Chiapas. Tercera edición. Consejo Estatal para la Cultura y las Artes de Chiapas, México.

Porter, T. A., y G. S. Wilkinson. 2001. Birth synchrony in greater spear-nosed bats (Phyllostomus hastatus). Journal of Zoology, London 253:383-390.

Racey, P. A., y A. C. Entwistle. 2000. Life history and reproductive strategies of bats. pp. 364-414. in Reproductive Biology of Bats (Crichton, E. G., y P. H. Krutzsch, eds.). Academic Press. London, Reino Unido.

Riechers-Pérez, A., M. Martínez-Coronel, y R. Vidal López. 2003. Consumo de polen de una colonia de maternidad de Leptonycteris curasoae de la cueva de los Laguitos, Chiapas, México. Anales del Instituto de Biología, UNAM, Serie Zoología 74:5366. 
Rojas-Martínez, A., A. Valiente-Banuet, M. C. Arizmendi, A. Alcántara-Eguren, y H. T. ARItA. 1999. Seasonal distribution of the long-nosed bat (Leptonycteris curasoae) in North America: does a generalized migration pattern really exist? Journal of Biogeography 26:1065-1077.

SEMARNAT. 2010. Norma Oficial Mexicana NOM-059. Protección ambiental-Especies nativas de México de flora y fauna silvestres-Categorías de riesgo y especificaciones para su inclusión, exclusión o cambio-Lista de especies en riesgo. Diario Oficial de la Federación, jueves 30 de diciembre.

Silva-Taboada, G. 1979. Los Murciélagos de Cuba. Editorial Academia. La Habana, Cuba.

Speakman, J. R., y D. W. Thomas. 2003. Physiological ecology and energetics of bats. pp. 430-490 in Bat Ecology (Kunz, T. H., y M. B. Fenton, eds.). The University of Chicago Press. Chicago, EE.UU.

Stern, A. A., Y T. H. Kunz. 1998. Intraspecific variation in postnatal growth in the greater spear-nosed bat. Journal of Mammalogy 79:755-763.

Stern, A. A., T. H. Kunz, y S. S. Bhatt. 1997. Seasonal wing loading and the ontogeny of flight in Phyllostomus hastatus (Chiroptera: Phyllostomidae). Journal of Mammalogy 78:1199-1209.

TAft, L. K., y C. O. Handeey, JR. 1991. Reproduction in a captive colony. Pp. 19-41 enDemography and natural history of the common fruit bat, Artibeus jamaicensis, on Barro Colorado Island, Panamá. (Handley, C. O. Jr., D. E. Wilson, y A. L. Gardner, eds.). Smithsonian Contribution to Zoology 511.

Tutrue, M. D. 1975. Population ecology of the gray bat (Myotis grisescens): factors influencing early growth and development. Occasional Papers of the Museum of Natural History, The University of Kansas 36:1-24.

Tuttle, M. D., y D. Stevenson. 1977. Variation in the cave environment and its biological implications. 108-121 in National Cave Management Symposium Proceedings. (R. Zuber, J. Vhester, S. Gilbert, y D. Rhoades, eds.). Adobe Press. Albuquerque, EE.UU.

Tuttle, M. D., y D. Stevenson. 1982. Growth and survival of bats. Pp 105-150 in The Ecology of bats (Kunz, T. H. ed.). Plenum Press. New York, EE.UU.

Wilkinson, G. S., y T. H. Fleming. 1996. Migration and evolution of lesser long-nosed bats Leptonycteris curasoae, inferred from mitochondrial DNA. Molecular Ecology 5:329-339.

Wyant, K. A., y R. A. Adams. 2007. Prenatal growth and development in the Angolan free-tailed bat, Mops condylurus (Chiroptera: Molossidae). Journal of Mammalogy 88:1248-1251.

ZAR, J. H. 1996. Biostatistical Analysis. 3th. Ed., Prentice Hall, New Jersey, EE.UU.

Zuluinger, E. M., R. E. Ricklefs, K. H. Redford, y G. M. Mace. 1984. Fitting sigmoidal equations to mammalian growth curves. Journal of Mammalogy 65:607-636. 
Sometido: 26 de noviembre de 2014

Revisado: 6 de marzo de 2014

Aceptado: 4 de abril de 2014

Editor asociado: Juan Pablo Gallo

Diseño gráfico editorial: Gerardo Hernández 